\title{
Design and Realization of a Novel Performance and Durability Test Platform for Anti-lock Braking Module
}

\author{
Chun-Hung $\mathrm{Wu}^{*}$, Shu-Heng Guo, Kuo-Sheng Chen \\ Department of Mechanical Engineering, National Cheng-Kung University, \\ No.1, Daxue Rd., East Dist., Tainan City 701, Taiwan \\ *Corresponding Author: jhwmusa@gmail.com
}

\begin{abstract}
Anti-lock braking systems (ABS) have been used for improving the vehicle safety in the past few decades. A performance and durability test system for ABS modules is necessary for guiding the product improvement. In this work, a novel versatile test system with a complete sensorial system is designed and realized for fulfilling the durability and performance test of ABS. Unlike traditional approach using wheels, this system relies on electrical signal to trigger the action of ABS. This results in a simple, compact, and versatile design. The test mainly concerns the performance of the hydraulic control unit (HCU) of ABS by examining the hydraulic responses after receiving the command. In addition, two commercial Bosch ABS modules are used on the system as the test samples for the purpose of validation and demonstration. In parallel, the results of the HCU performance test are also compared with that obtained from a commercial test platform based on the same ABS unit. The test results are similar and this fact demonstrates the applicability of the designed system. In comparison with the corresponding commercial system, the designed system is relatively low cost, size compact, and more versatile in testing manner. In the future, more sensors and functions would be added to enhancing the functionality and reliability of the system and becomes compatible with a traditional electronic control unit (ECU) performance test.
\end{abstract}

Keywords: anti-lock braking system, hydraulic control unit, performance test, durability test.

\section{Introduction}

Anti-lock braking systems (ABS) have been widely mounted in many types of vehicles in the past few decades ${ }^{(1)}$ and many test systems had been designed in other works ${ }^{(1-4)}$ for characterizing the braking performance of ABS-mounted car. However, those systems are designed for addressing braking dynamics, not for testing the ABS units. That is, those test systems focus on their designs of control strategies and are not for evaluating the performance and durability of the ABS modules themselves. For example, J.A. Cabrera et al. built up a versatile tire testing machine acquiring the data of braking tires with simulated ABS system ${ }^{(5)}$ as the focus of their work. However, it is mainly suitable for studying braking performance but not for the performance and durability test because this paper focuses only on the tire speed, slip ratio, slip angle, etc. The performance and durability test on ABS modules itself should be a necessary part for a complete ABS design and producing cycle. As a result, a test system focused on the ABS module itself only is important and cannot be replaced by others in product development.

Hydraulic control unit (HCU) is the actuator of the system $^{(6)}$. The function of pressure modulation of an HCU is greatly affected by the design of the hydraulic circuit and the bandwidth of the solenoid valve. It is apparently that the performance of an $\mathrm{HCU}$ will determine the function of an ABS system. There are several standard tests in the HCU production process in industry ${ }^{(7)}$. Therefore, the performance of the $\mathrm{HCU}$ must also considered when one is testing an ABS. However, it is unrealistic for researchers to adopt a commercial test system because of its high price and nonversatile design.

In general, it is indispensable for ABS manufacturers and researchers to test their new prototypes or compare it with other successful products with a reliable test system. Furthermore, very few research works published regarding the designs and realizations of ABS test systems. In addition, an ABS test system with multiple purposes, such as durability test and HCU performance test, is usually not 
affordable for small businesses or university laboratories.

Motivated by the above needs, the goal of this work is to realize a novel versatile test system with both performance and durability tests for ABS modules. In addition, unlike commercial ones, this system is relatively compact, low-cost, and efficient. For the performance test, both the HCU actuating and the HCU module and entire ABS module with electronic control unit (ECU) performance tests are considered. For the durability test, the ABS module is triggered by a virtual wheel speed signals generated in host computer to ensure a long-term operation. Without the need of wheels and their associated driving system, this system represents a great saving in both space and cost and may be an appropriate test environment for both laboratory and related industries. the system design for $\mathrm{HCU}$ performance and ABS durability tests using ABS modules manufactured by Bosch $\mathrm{GmbH}$. The traditional ECU performance test which mainly concerns with controller ability will be established in the future.

The research flow of this work is shown in Fig.1. In Chapter 2, the concept of the entire test system and subsystems are introduced. It explains the relationship and integration between them. After that, the designs of the subsystems are presented in Chapter 3, followed by the integration and realization of the test systems presented in Chapter 4. In Chapter 5, serval HCU testing results for commercial ABS modules are presented for demonstrating the feasibility. In Chapter 6, essential discussion to address the near term future work are also provided for highlight the future perspective.

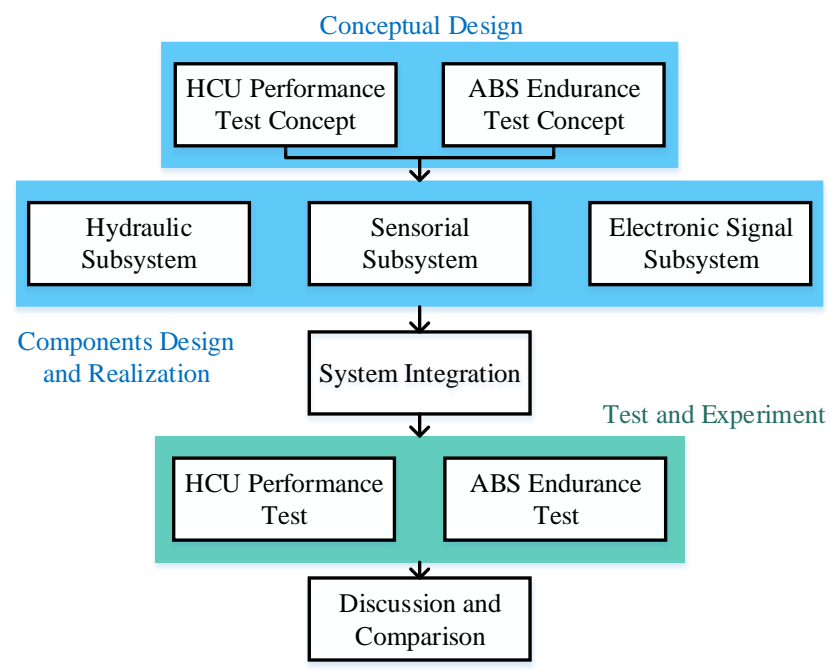

Fig. 1. Research flow.

\section{Conceptual Design}

As mentioned in the Chapter 1, the system designed would like to fulfill both the HCU performance and ABS durability tests. For carrying the HCU performance test, instead of sending the command directly from ECU, the solenoid valves of an HCU would be actuated by our own controlled current signals. The high pressure brake fluid provided by a high pressure source would then be modulated by the HCU and the dynamic response of fluid pressure are then obtained by the associated pressure sensors. By analyzing the sensor readouts, it is possible to find the dynamic characteristics in pressure buildup and release such as the rising time and pressure holding levels. The advantage of this design is that the actuation completely skips the ECU and the associated Controller Area Network (CAN) bus. This is important because that the control codes of ABS are usually not released. In short, this "dummy" test hires a host computer to generate desired command signals for the HCU and worked as a fake ECU. On the other hand, for the ABS durability test, the entire ABS module is triggered by virtual wheel speed signals generated by a special circuit instead of using real rotating wheels. Again, this fake wheel signals also bring convenience and flexibility for the testing.

Since both of test share almost the same major components, the main idea of this work is to design a single test system to fulfill both tests. Therefore, a switchable hydraulic circuit is designed in this work and is shown in Fig. 2 (a). There are two pressure sources in the system, including the hydraulic pump and the brake levers of a motorcycle. The source brake fluid passes through the manifold block with pressure sensors installed and flows to the ABS modules. In this work, two Bosch ABS modules are chosen for performing the HCU performance and ABS durability tests. The pressurized brake fluid passes through the ABS modules then returns to the manifold block, where pressure sensors are installed for sensing the return pressure. The fluid then flows to the wheel cylinders or nowhere.

For example, in the HCU performance test, the pressure generated by the pump passes through the HCU module actuated by current signals (dummy signals) Similarly, in the ABS durability test, the pressure generated by the brake levers pass through the ABS module triggered by virtual wheel speed signals. Pressures before and after ABS modules in two test are measured both. With this design, one can switch the pressure sources, ABS modules, and output loading at any time. That is, multiple tests can be realized on this test system.

In addition, a traditional drum type ECU performance test system can also be combined with this system, as can be 
illustrated in Fig. 2 (b). It indicates the extensibility of the system because of the completeness of the pressure sources and sensors. This part is important for control strategies of an ECU in ABS studies and it is in progress and will be completed in future.

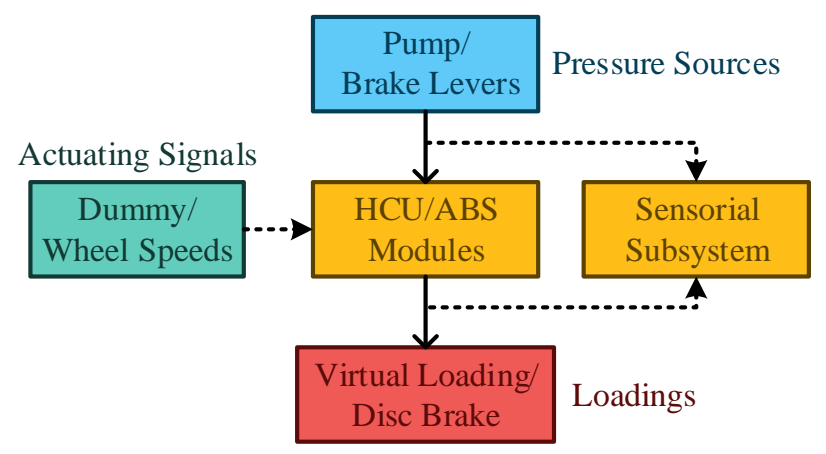

(a) Overall conceptual design.

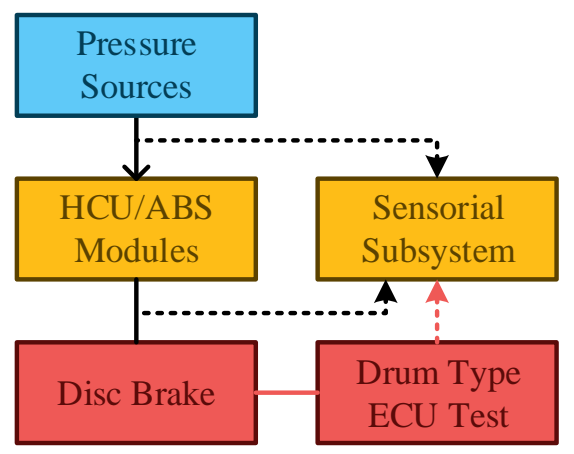

(b) Combination with drum type ECU test system.

Fig. 2. System structure diagram.

\section{Components Design}

\subsection{Hydraulic Subsystem}

For achieving both test as explained previous, high pressure valves are assembled in T-fittings as shown in Fig. 3 (a). Thus, one can switch the pressure sources and ABS modules at any time according to different types of tests. A stable and 120 bar pressure is needed for the HCU performance test as an industrial test criterion ${ }^{(7)}$. Therefore, a hydraulic pump with a maximum pumping capacity of 250 bar with a pressure regulator is chosen and is shown in Fig. 3 (b). For the ABS durability test, a for pushing the brake levers repeatedly is built to mimic the operation by a motorcyclist. This subsystem is shown in Fig. 3 (c). The brake levers are pushed by linear motors driven by an NI myRIO signal processing unit. Furthermore, because of the

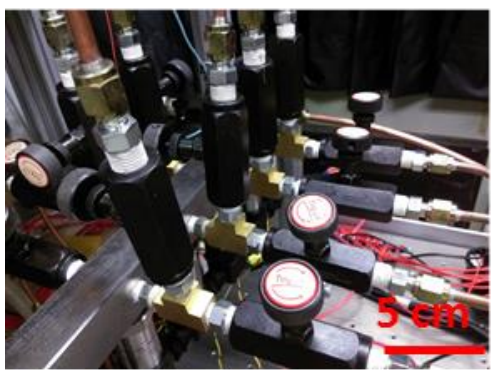

(a) High pressure valves.

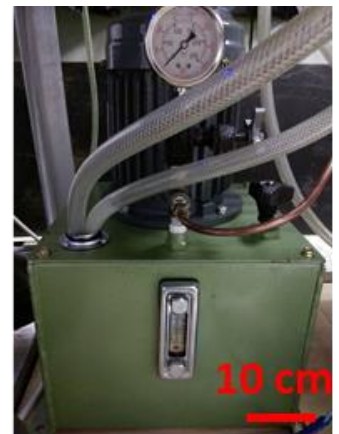

(b) Hydraulic pump.

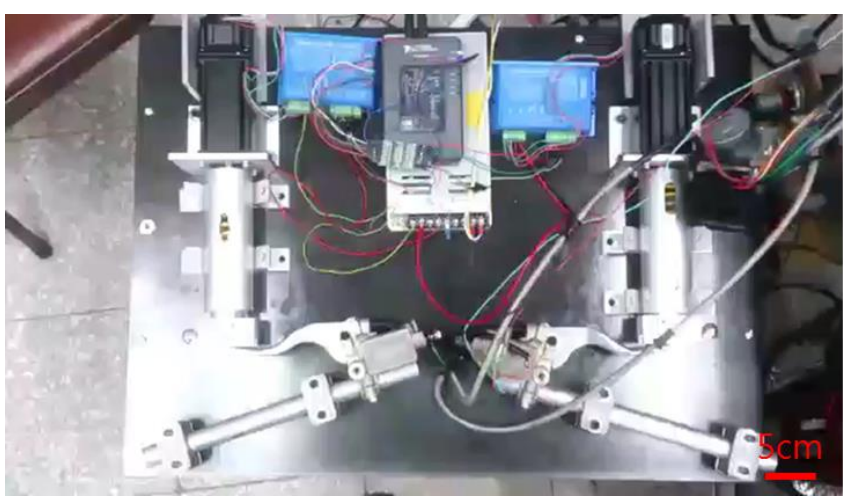

(c) Linear-motor-driven brake levers subsystem.

Fig. 3. Components details.

high pressure and high-repeat testing condition, copper tubes but not hoses are chosen for minimizing the undesirable compliance for maintaining high fluid pressure.

\subsection{Sensorial Subsystem}

The pressure sensors, BCM $131 \mathrm{~F}$ with measuring range 0 bar to $250 \mathrm{bar}$, are chosen in this work and installed on a manifold block. They record inlet and outlet brake fluid pressures of the ABS modules so one can obtain pressure modulations and acting histories which are interested in durability test. The vibration signals are also important in durability test since they may indicate the break down and even show early warning of the ABS. Meanwhile, braking performance of a vehicle is significantly affected by the temperature rise of the brake fluid and high temperature may cause braking fault ${ }^{(8)}$. As a result, essential fluid temperature measurement is required. So far the manifold block with 6 pressure sensors is completed and they are shown in Fig. 4. The vibration and temperature sensors will be incorporated in the near future. Nevertheless, the preliminary demonstration of vibration measurement during operation of the ABS is illustrated and discussed in Chapter 5 for highlight its importance. 


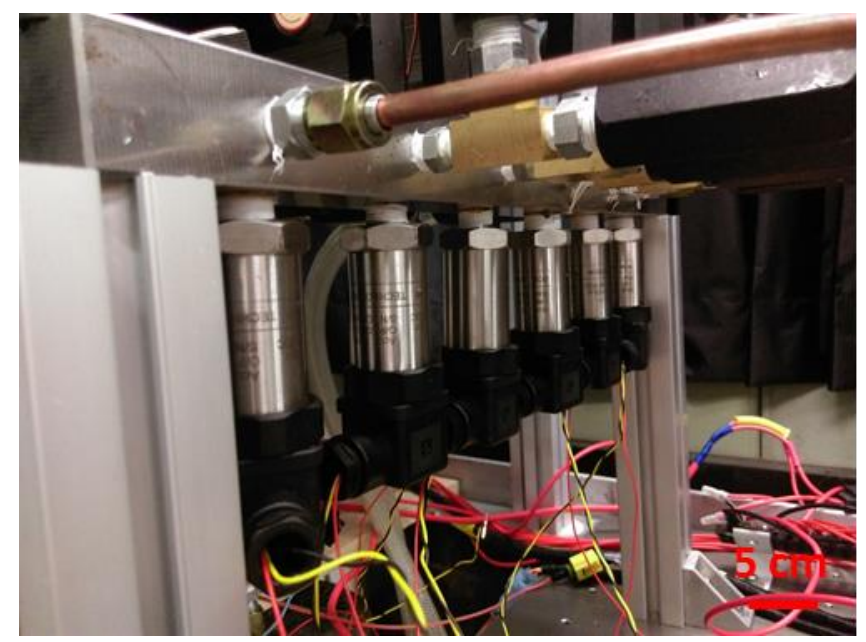

Fig. 4. Pressure sensors in the manifold block.

\subsection{Electronic Signal Subsystem}

As mentioned earlier, instead of using physical hardware, electrical signals are generated to triggered the HCU and ABS modules in this work. In the HCU performance test, the solenoid valves and motor need to be actuated. Since one cannot obtain the messages across the CAN to command them by the ECU unless the signal layout of the bus is fully released by the manufacturer. Therefore, as shown in Fig. 5, the ECU is completely ignored in this case. To do so, the pins of the solenoid valves and motor are directly connected to the host electronics for receiving command signals. Meanwhile, in the ABS durability test, the fake wheel speed signals generated are sent to the ECU to triggered the ABS action.

In order to faithfully create the triggering signal, the output of wheel speed sensors mounted on a wheel is analyzed since it provides real signals for actuating ECU. The output current of the wheel speed sensor is a pulsing signal, where the wheel speed can be determined by counting the pulse frequency. This pulsing signal is then converted into a current between 7 and $14 \mathrm{~mA}$ before entering the ECU. As a result, a corresponding signal generator is designed to generate desired pulse signal (for mimic wheel rotation) and then converted to the corresponding current signal for triggering ECU. By this approach, the ECU operates as if it is mounted on a motorcycle.

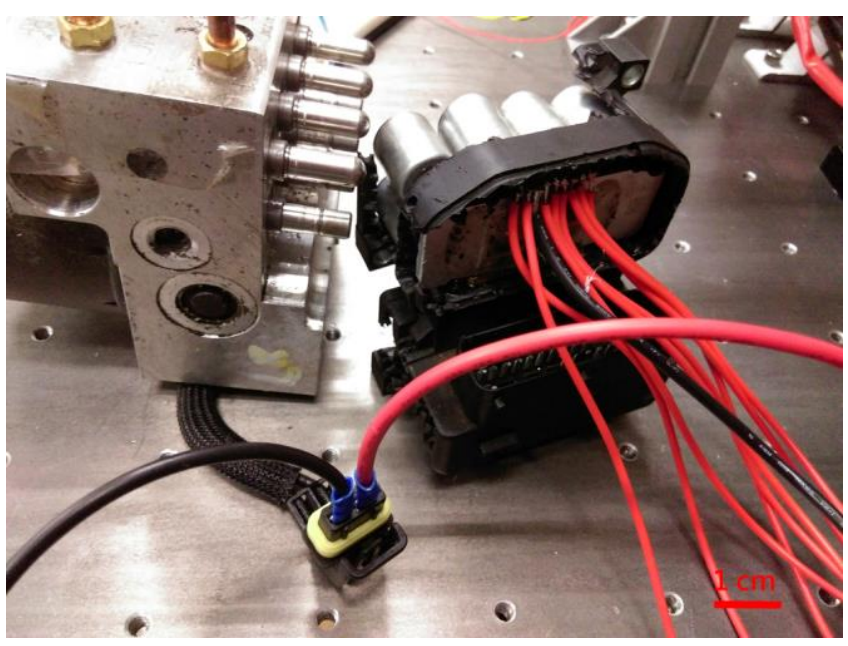

Fig. 5. Dummy Signals Connection.

\section{System Integration}

The test system is established and shown in Fig. 6. The hydraulic pump and brake levers pump the brake fluid to the ABS modules and the fluid pressure is monitored by the pressure sensors installed at the manifold block. A Bosch ABS HCU of a Honda compact car is chosen for the HCU performance test and a Bosch ABS Generation 9 of a PGO light motorcycle is for the ABS durability test. After hydraulic modulating process in the ABS modules, the brake fluid returns to manifold block and its pressure is monitored. An extra disc brake assemblies and drum type ECU test subsystem will be added in the future. The control panel is responsible for power up, overload monitoring, and emergency stop. In addition, a speed meter of motorcycle is also assembled on the system for indicating the speed of virtual wheel speed signal generated by this work works. Furthermore, an NI myRIO and DAQ (NI-9205) are used to convert digital command to analog and acquire the data of sensors, respectively.

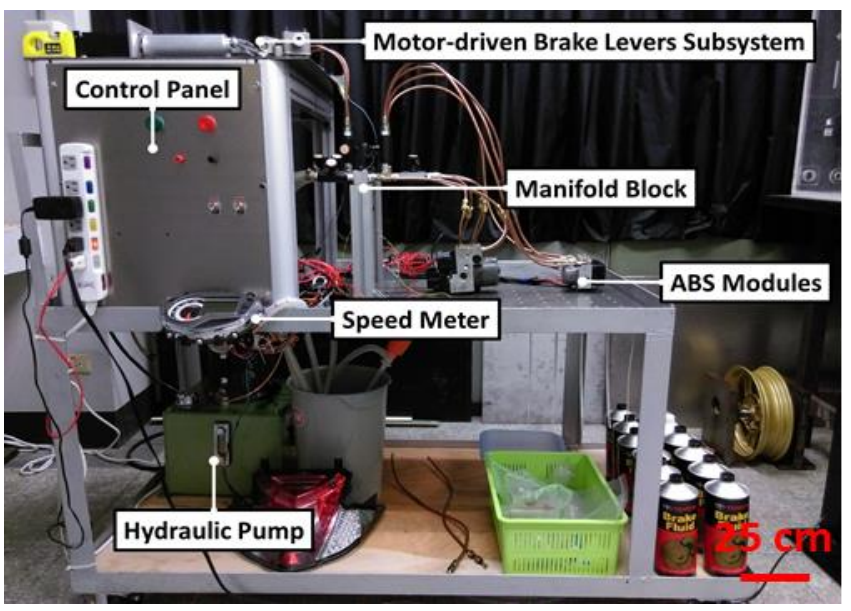

(a) Overall view. 


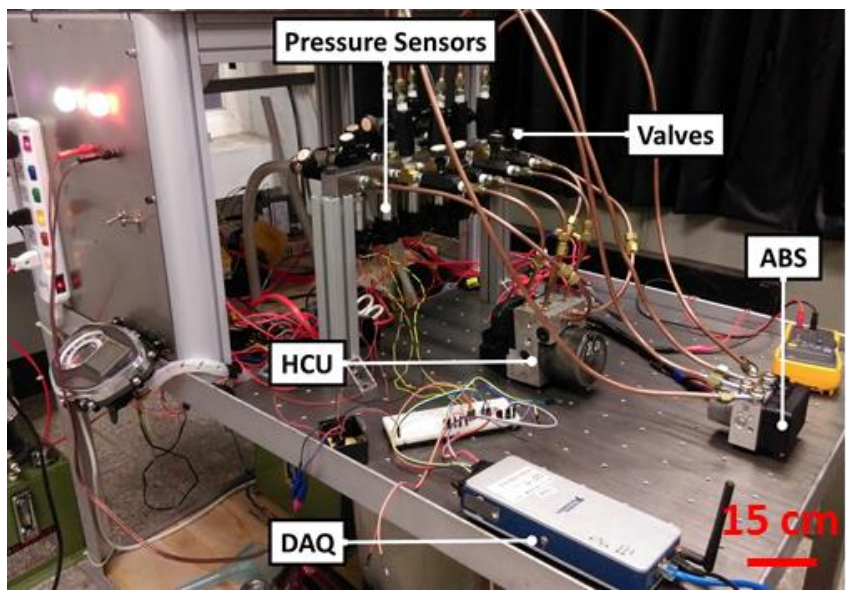

(b) Enlarged view.

Fig. 6. The designed ABS test system.

\section{Demonstrations}

In this chapter, serval tests are presented as application examples for demonstrating the performance and feasibility of this system. Fig. 7 is a typical test result for pressure building up of the linear-motor-driven brake levers subsystem. It shows that the pressure rises when the brake lever is pushed and drops when the lever released. Such a process will be repeated in the future for the durability test and the life can be estimated.

Furthermore, two HCU performance tests, namely pressure buildup and external sealing tests, are shown in Fig. 8 . In the pressure buildup test, the inlet valves closed by the dummy signal, and then a pressure generated by the hydraulic pump for representation of the supplying of a master cylinder (MC); after the pressure of master cylinder reaching stable, inlet valves are opened one by one and thus pressures of respective wheel cylinder circuit build up. The standard test pressure is $120 \mathrm{bar}^{(7)}$, but for the purpose of demonstration, 95 bar is presented and only two pressures of wheel cylinders is shown in this article. In the second subplot in Fig. 8 (a), the history of the pressure buildup of the rear left wheel cylinder (RL) is shown. The pressure rises rapidly at the middle process and slows down when it closes to the target pressure. To indicate the performance, one calculates the maximal pressure buildup rate defined as ${ }^{(7)}$ :

$$
\dot{p}_{\max }=\frac{p_{2}-p_{1}}{t_{2}-t_{1}}
$$

where $p_{1}$ and $p_{2}$ are, respectively, the closet pressure to 20 bar and $80 \mathrm{bar}$, which is the range of the fastest pressure buildup curve, and $t_{1}$ and $t_{2}$ are the closet time to 20 bar and 80 bar, respectively. In this case, the maximal pressure buildup rate of rear left wheel cylinder is $9447 \mathrm{bar} / \mathrm{s}$.

In the static external sealed test, the outlet valves are opened after the master cylinder supplies pressurized brake fluid and thus whole HCU circuit is full of high pressure brake fluid for a few seconds. In Fig. 8 (b), the pressures drop and then rise again immediately. One can obtain its seal from calculating the maintaining of the pressure curves. In Fig. 9, the vibration signal obtained by using the accelerometer equipped in MyRIO module on the Bosch ABS 9. It briefly shows the vibration of the ABS when it is acting and can be used for future device status monitoring purpose.

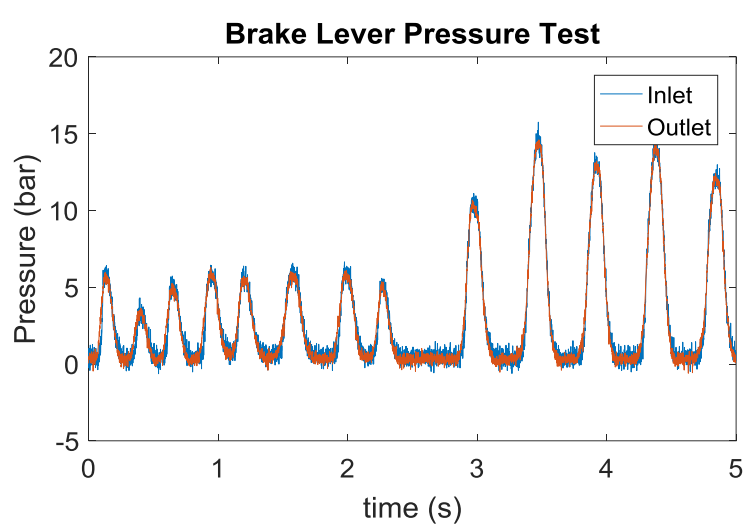

Fig. 7. Pressure buildup by brake lever.
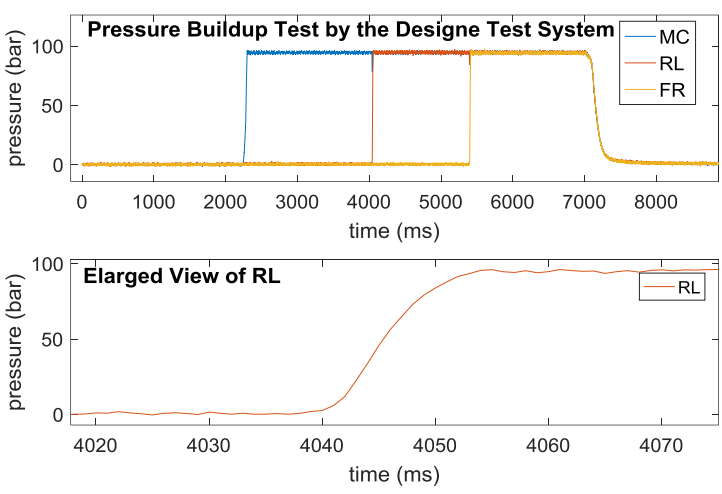

(a) Pressure buildup test. 


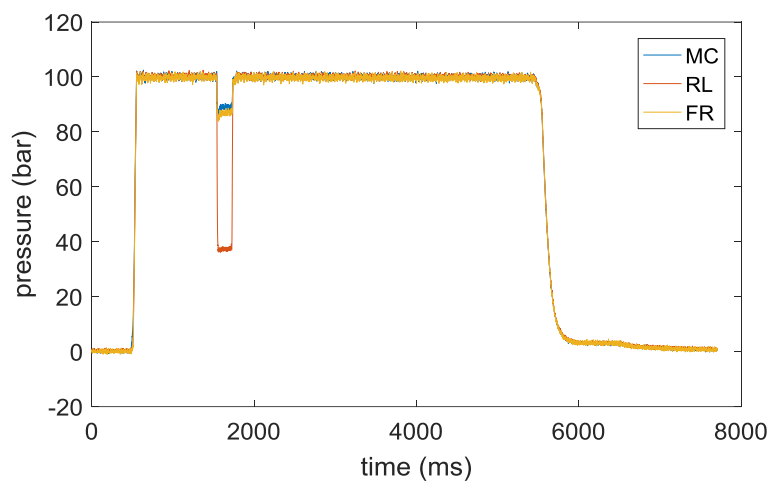

(b) Static external sealed test.

Fig. 8. HCU performance test demonstration.

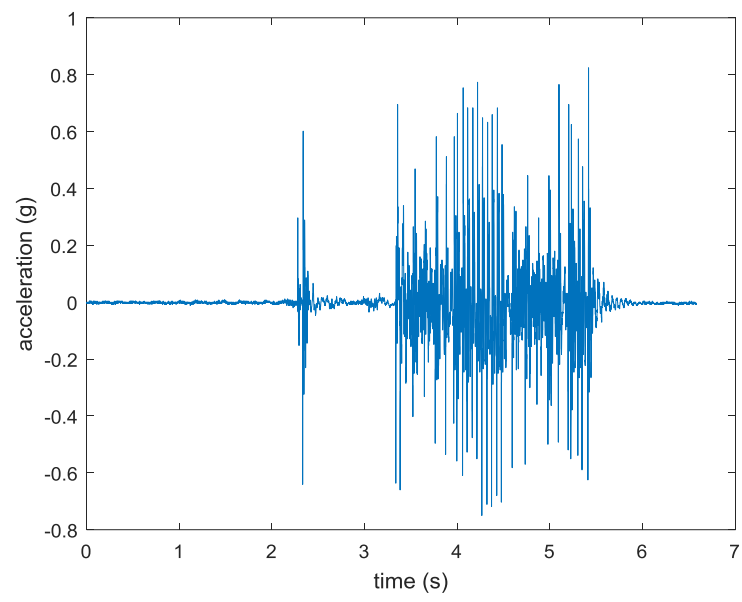

Fig. 9. Acceleration signal of ABS.

\section{Discussion and Future Work}

Chapter 5 shows the capability to build up and sense hydraulic pressure. However, the signal is a little bit noisy and therefore it needs to be improved by better shielding or applying proper signal processing, such as moving average or low-pass filter. The linear-motor-driven brake levers subsystem has built pressure successfully and the next task is to program it carefully for synchronizing their movement with the virtual wheel speed signals for the ABS durability test. From the test data, an online monitoring or diagnostic system can be established for examining the possible physical failure such as oil leakage.

It is also interesting to make a brief comparison between the designed system and a commercial one (HT-SAAE). The test results are shown in Fig. 10(a). By comparing with the test results shown in Fig. 8 (a), the pressure buildup responses are similar. However, the pressure rising at the just beginning point is steeper and the pressure rising rates are faster. It is believed that these differences are caused by different power source characteristics. However, the balance between left and right wheel cylinders is mainly concerned ${ }^{(7)}$. That is, although the pressure rising rates are different, the pressure difference between left and right wheel cylinders are identity in the two test systems.

Meanwhile, the static external sealed test on the commercial test platform is shown in Fig. 10 (b). By comparing with Fig. 8 (b), again, the test results are in general similar. However, the pressures in commercial test system drop and hold without a recovering. They should rise to the target pressure as done in this work shown in Fig. 8 (b) and this situation has been confirmed as a design fault by its manufacturer. More tests with more wheel cylinder circuits will be finished in the near term future. Furthermore, the acceleration signal is monitored for the durability of ABS. Accelerometer and thermal sensors will also be added on in the future.
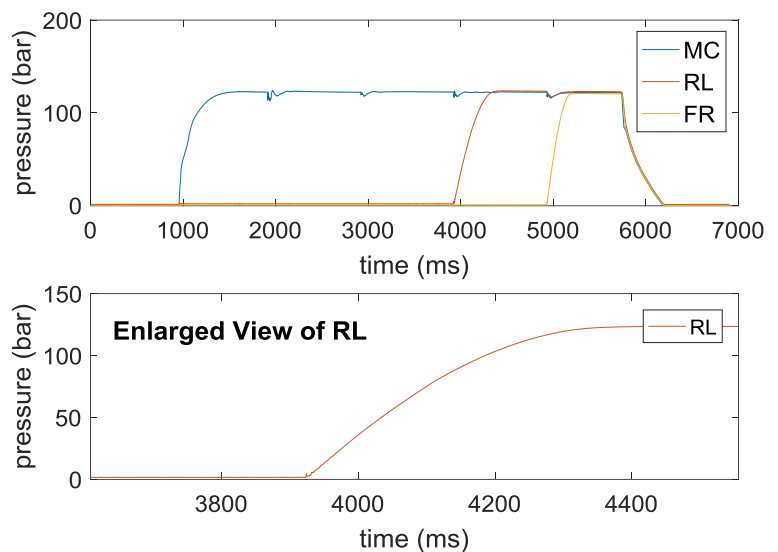

(a) Pressure buildup test

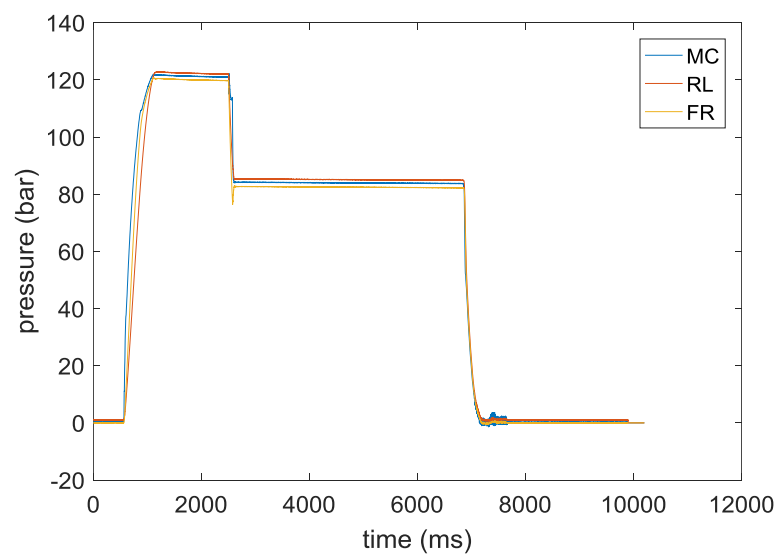

(b) Static external sealed test.

Fig. 10. HCU performance test in a commercial test system.

\section{Conclusion}


A well-behaving performance and durability test system is critical for developing ABS module in their product process cycle. A novel, compact, and low cost versatile test system with complete sensorial subsystem is designed and realized in this work. ABS durability and HCU performance can be tested using this system. The initial test and demonstration are then conducted by hiring the commercially available Bosch HCU and ABS modules. By comparing the test results with a commercial test system, it is concluded that the system can achieve similar testing performance obtained by the commercial test platform. In the future, more sensors will be incorporated and the system will be further modified to enhance the reliability and the quality of sensed signals. An ECU performance test is also underway for demonstrating the versatility of the designed system.

\section{Acknowledgments}

This research is supported by the Ministry of Science and Technology in Taiwan, ROC under the contract number 105-2221-E-006-100-MY and Yen Tjing Ling Industrial Development Foundation. The comments and assistances from Prof. S.-F. Ling and Mr. C.-E. Wu from Lioho Machine Works, Ltd are great appreciated.

\section{References}

(1) Ming-chin Wu and Ming-chang Shih : "Simulated and experimental study of hydraulic anti-lock braking system using sliding-mode PWM control", Mechatronics, Vol. 13, No. 4, pp. 331-351, 2003

(2) Yesim Oniz, Erdal Kayacan, and Okyay Kaynak : "A Dynamic Method to Forecast the Wheel Slip for Antilock Braking System and Its Experimental Evaluation", IEEE Transactions on Systems, Man, and Cybernetics, Part B (Cybernetics), Vol. 39, No. 2, pp. 551-560, 2009

(3) Seongho Choi and Dong-Woo Cho : "Design of Nonlinear Sliding Mode Controller with Pulse Width Modulation for Vehicular Slip Ratio Control", Vehicle System Dynamics, Vol. 36, No. 1, pp. 57-72, 2001

(4) Juan A. Cabrera, Antonio Ortiz, Juan J. Castillo, and Antonio Sim'on : “A Fuzzy Logic Control for Antilock Braking System Integrated in the IMMa Tire Test Bench", IEEE Transactions on Vehicular Technoloogy, Vol. 54, N0. 6, 2005

(5) J.A. Cabrera , A. Ortíz , A. Simón , F. García and A. Pérez La Blanca : “A Versatile Flat Track Tire Testing
Machine”, Vehicle System Dynamics, Vol. 40, No. 4, pp. 271-284, 2003

(6) Wei Liu, Wenying Liu, and Haitao Ding : "Hydraulic Control Unit Modeling of Electronic Stability Program Based on AMESim", Applied Mechanics and Materials, Vol. 133-134, pp. 402-407, 2012

(7) Lioho Machine Works, Ltd. : "The User Manual for HCU Testing System", n. d.

(8) F. Talati \& S. Jalalifar, "Analysis of heat conduction in a disk brake system", Heat Mass Transfer, Vol. 45, pp. 1047-1059, 2009 